\title{
Coffee drinking and cancer risk: an umbrella review of meta-analyses of observational studies
}

\author{
Long-Gang Zhao ${ }^{1}$, Zhuo-Ying Li ${ }^{1}$, Guo-Shan Feng ${ }^{1}$, Xiao-Wei Ji ${ }^{1}$, Yu-Ting Tan ${ }^{1}$, Hong-Lan Li ${ }^{1}$, Marc J. Gunter ${ }^{2}$ and \\ Yong-Bing Xiang ${ }^{1 *}$ (1)
}

\begin{abstract}
Background: Epidemiological studies on the association between coffee intake and cancer risk have yielded inconsistent results. To summarize and appraise the quality of the current evidence, we conducted an umbrella review of existing findings from meta-analyses of observational studies.

Methods: We searched PubMed, Embase, Web of Science and the Cochrane database to obtain systematic reviews and meta-analyses of associations between coffee intake and cancer incidence. For each association, we estimated the summary effect size using the fixed- and random-effects model, the 95\% confidence interval, and the 95\% prediction interval. We also assessed heterogeneity, evidence of small-study effects, and excess significance bias.

Results: Twenty-eight individual meta-analyses including 36 summary associations for 26 cancer sites were retrieved for this umbrella review. A total of 17 meta-analyses were significant at $P \leq 0.05$ in the random-effects model. For the highest versus lowest categories, 4 of 26 associations had a more stringent $P$ value $\left(P \leq 10^{-6}\right)$. Associations for five cancers were significant in dose-response analyses. Most studies (69\%) showed low heterogeneity $\left(I^{2} \leq 50 \%\right)$. Three and six associations had evidence of excessive significance bias and publication bias, respectively. Coffee intake was inversely related to the risk of liver cancer and endometrial cancer and was characterized by dose-response relationships. There were no substantial changes when we restricted analyses to meta-analysis of cohort studies.
\end{abstract}

Conclusions: There is highly suggestive evidence for an inverse association between coffee intake and risk of liver and endometrial cancer. Further research is needed to provide more robust evidence for cancer at other sites.

Keywords: Coffee, Cancer, Umbrella review, Grading evidence, Observational studies

\section{Background}

Coffee, a beverage prepared using roasted beans from berries of the coffee plant, is one of the most widely consumed beverages in the world. Given the popularity of coffee drinking, any benefits of coffee on human health may exert substantial public health effects. Therefore, investigations of coffee intake and its potential health effects have been an area of research interest [1]. These potential links with health outcomes, especially with

\footnotetext{
* Correspondence: ybxiang@shsci.org

${ }^{1}$ State Key Laboratory of Oncogene and Related Genes \& Department of Epidemiology, Shanghai Cancer Institute, Renji Hospital, Shanghai Jiaotong University School of Medicine, Shanghai, China

Full list of author information is available at the end of the article
}

cancers, are appealing to the policy maker and epidemiologists, as well as to the public.

Researchers have been investigating the associations between coffee consumption and the risk of cancer for decades [2, 3]. Biochemical studies have indicated that hundreds of biologically active compounds including caffeine, flavonoids, lignans, and other polyphenols are found in roasted coffee [4]. These coffee-derived compounds have been shown to increase energy expenditure, regulate DNA repair-related genes, and inhibit the chronic inflammatory response in animal studies [5]. However, human studies from different regions or populations have generated inconsistent results. Early studies on the impacts of coffee mainly focused on bladder

(c) The Author(s). 2020 Open Access This article is distributed under the terms of the Creative Commons Attribution 4.0 International License (http://creativecommons.org/licenses/by/4.0/), which permits unrestricted use, distribution, and reproduction in any medium, provided you give appropriate credit to the original author(s) and the source, provide a link to the Creative Commons license, and indicate if changes were made. The Creative Commons Public Domain Dedication waiver (http://creativecommons.org/publicdomain/zero/1.0/) applies to the data made available in this article, unless otherwise stated. 
cancer, which indicated a higher risk of bladder cancer based on case-control studies [6,7]. Studies conducted in the past 30 years have suggested a potential relation between coffee consumption and cancers of the liver, endometrium, and melanoma [8-11]. Even though numerous findings have accumulated to support the benefits of recommendations on coffee drinking daily habits for cancer prevention, there is no convincing evidence because of inconsistent results across studies and issues with data quality. Previous studies focused on all kinds of health outcomes rather than only cancer at various sites $[12,13]$. It is therefore important to summarize the current epidemiological evidence and appraises its quality based on a comprehensive criteria of grading evidence.

Umbrella reviews offer the possibility to understand the strength of evidence and the extent of potential biases for the associations between coffee and cancer incidence [14]. In this report, we summarized the epidemiological evidence on associations between coffee consumption and the risk of developing any type of cancer. We described the magnitude, direction, and significance of the observed associations between coffee intake and cancer incidence. We also evaluated whether there is evidence of biases in the findings and identify the most robust associations which have fewer potential biases.

\section{Methods}

We had registered this protocol within the PROSPERO database for systematic reviews and meta-analyses (registration number: CRD42017084381). The protocol has been designed and reported according to the reporting guidance provided in the Preferred Reporting Items for Systematic Reviews and Meta-Analyses Protocols (PRISMA-P) statement [15].

\section{Literature search, study selection, and data extraction}

Three researchers independently searched the PubMed, Embase, Web of Science and the Cochrane database of systematic reviews from inception to February 2019 for meta-analyses or systematic reviews of observational studies investigating the association between coffee consumption and risk of any developing cancer. We screened the titles and abstracts of all articles and then examined in detail and screened for applicability through full text. Further information on screening, selection procedure, and data collection were provided in the Additional file.

\section{Data analysis}

We carried out a descriptive analysis of systematic reviews. The data from each systematic review and findings based on methodological quality were used to build evidence tables. We reestimated the summary effect size and its 95\% confidence interval $(95 \% \mathrm{CI})$ [16]. In the sensitivity analysis, we reselected meta-analysis with the largest number of only cohort studies from the database. We evaluated heterogeneity by estimating the variance between studies using Cochran's Q test and the $\mathrm{I}^{2}$ statistic $[17,18]$. We also estimated the $95 \%$ prediction interval (95\%PI) [19]. An indication of small study effects was evaluated based on the Egger's test [20]. We assessed excess significance bias by evaluating whether the observed number of studies with nominally statistically significant results was different from the expected number of studies with statistically significant results [21]. Details can be found in the Additional file 1 .

\section{Grading the evidence}

We categorized the associations between measures of coffee consumption and cancers into strong, highly suggestive, suggestive, weak, or no association depending on the strength and validity of the evidence, such as $P$ value of the random-effects model, total cases, $\mathrm{I}^{2}$ statistic of heterogeneity, $95 \% \mathrm{PI}$, small study effects, and excess significance bias [22-24]. Details were provided in Table 3.

All statistical analyses were performed using Stata version 14.0 (Stata Corp LP, College Station, TX, USA). A two-sided $P$ value less than 0.05 was considered as reaching statistical significance if not specified.

\section{Results}

\section{Literature selection}

The flow chart of article selection was provided in Fig. 1. Briefly, we identified 272 articles after removing the duplicates. We provided a list (online Additional file 1: Table S1) for excluded studies that did not meet our inclusion criteria.

After two steps of careful reviews, we identified 28 original meta-analyses that investigated associations between coffee intake and cancer incidence. Among these studies, we finally selected 36 non-overlapped associations from 28 independent original studies. Twenty-six of these used the highest versus lowest intake of coffee drinking in a categorical and the remainder analyzed the data in a dose-response analysis.

\section{Characteristics of meta-analyses included in this umbrella review}

Table 1 summarizes these 36 independent associations including 26 cancer sites. The median number of individual studies for each cancer was 15 , ranging from 4 to 54 . The number of cases ranged from 255 to 104,770. All except for three comparisons (acute myelogenous leukemia, biliary tract cancer, and glioma) included more than 1000 cases in the meta-analyses. Of 448 individual studies for the highest versus lowest comparison, 268 (59.8\%) were 


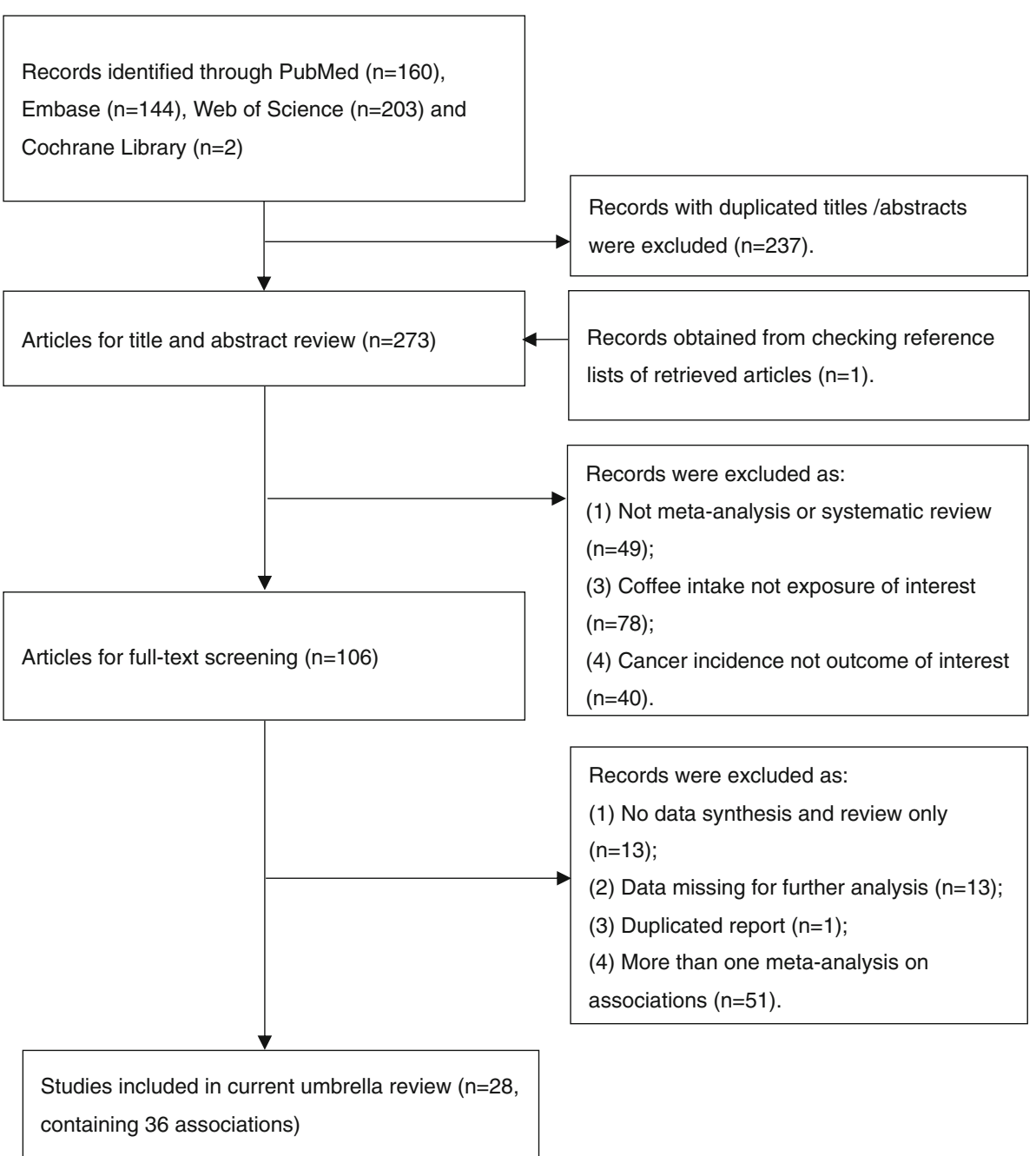

Fig. 1 Flow diagram of the selection process of meta-analyses on coffee intake and cancers

case-control studies and 179 (40.0\%) were cohort studies and one was a cross-sectional study. Of 144 individual studies for dose-response analysis, 40 (27.8\%) were casecontrol studies and 104 (72.2\%) were cohort studies.

For associations with more than one published meta-analysis, there was an agreement in general on the direction, magnitude, and significance of the summary associations given the different numbers of studies included. Details were provided in the online Additional file 1: Table S1.

\section{Evidence summary}

The summary random-effects estimates were significant at $P \leq 0.05$ in 17 meta-analyses (39\%). For the highest versus lowest categories $(\mathrm{H} / \mathrm{L})$, four of 26 associations had a more stringent $P$ value $\left(P \leq 10^{-6}\right)$. Overall, coffee intake was associated with incident bladder cancer, endometrial cancer, liver cancer, and oral cancer in the highest versus lowest analyses. Coffee was associated with five cancers in dose-response analyses (cancers of breast, colon, endometrium, liver, and lung). There is a slight difference between the random-effects model and fixed-effects model. The studies with the smallest standard error of each association suggested that 16 of 36 were significant at $P=0.05$.

The $\mathrm{Q}$ test showed significant heterogeneity $(P \leq 0.10)$ for 11 (31\%, 11/36) meta-analyses (Table 2 and Fig. 2). Most studies $(69 \%, 25 / 36)$ showed low heterogeneity $\left(\mathrm{I}^{2} \leq 50 \%\right)$. The associations showed substantial heterogeneity $\left(\mathrm{I}^{2}>75 \%\right)$ included laryngeal cancer $(\mathrm{H} / \mathrm{L})$ and lung cancer $(\mathrm{H} / \mathrm{L})$. Only three associations (acute lymphocytic leukemia, endometrial cancer, and liver cancer) had a 95\% PI that excluded the null value. Details were provided in the Additional file 2.

\section{Excess significance test and publication bias}

From Table 2, the evidence for excess significance using the largest study estimate as the plausible effect size was 


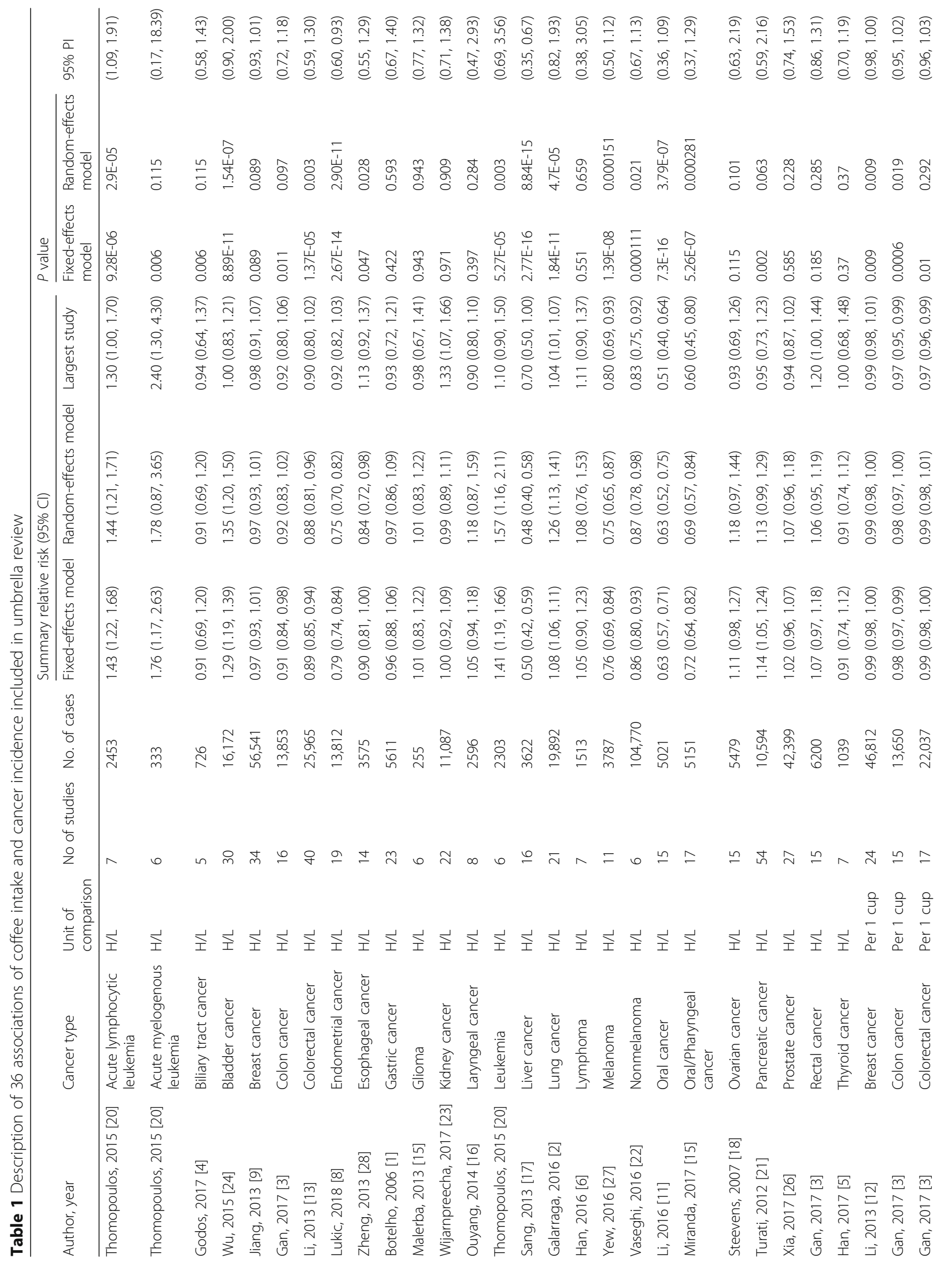




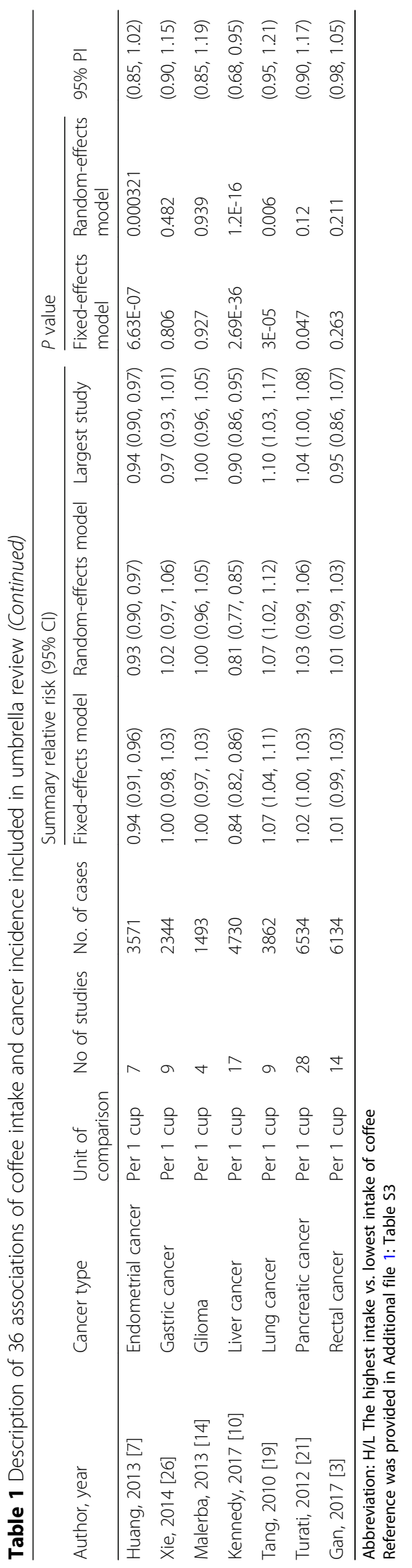




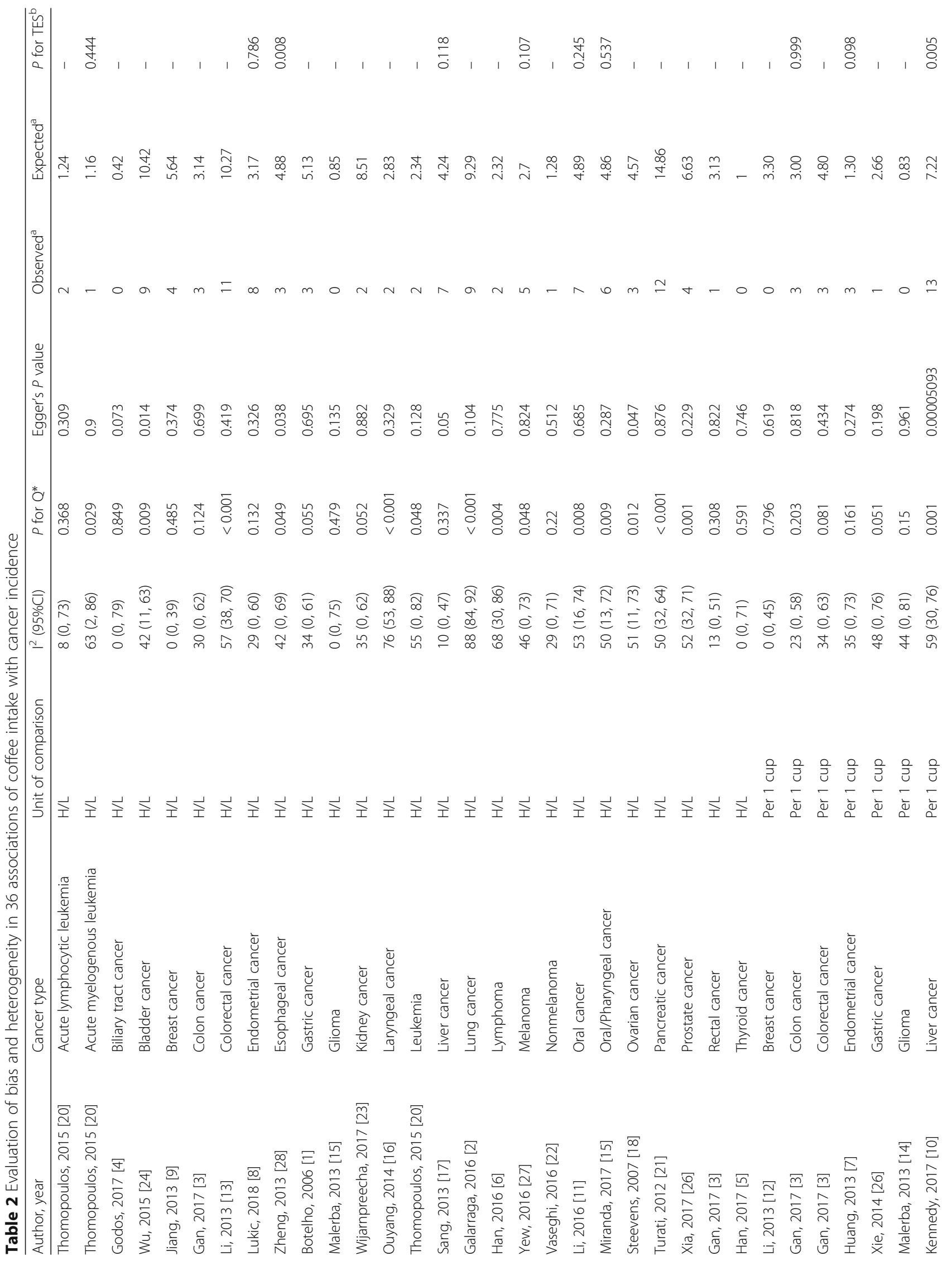




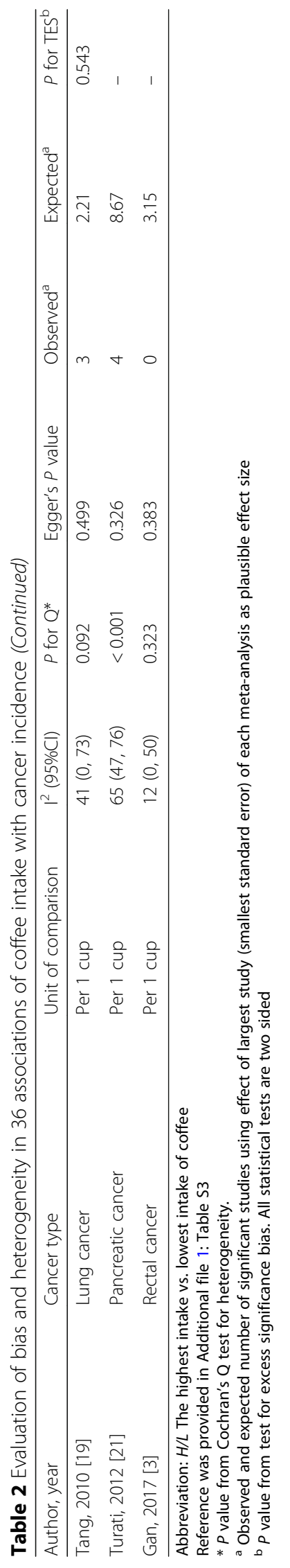




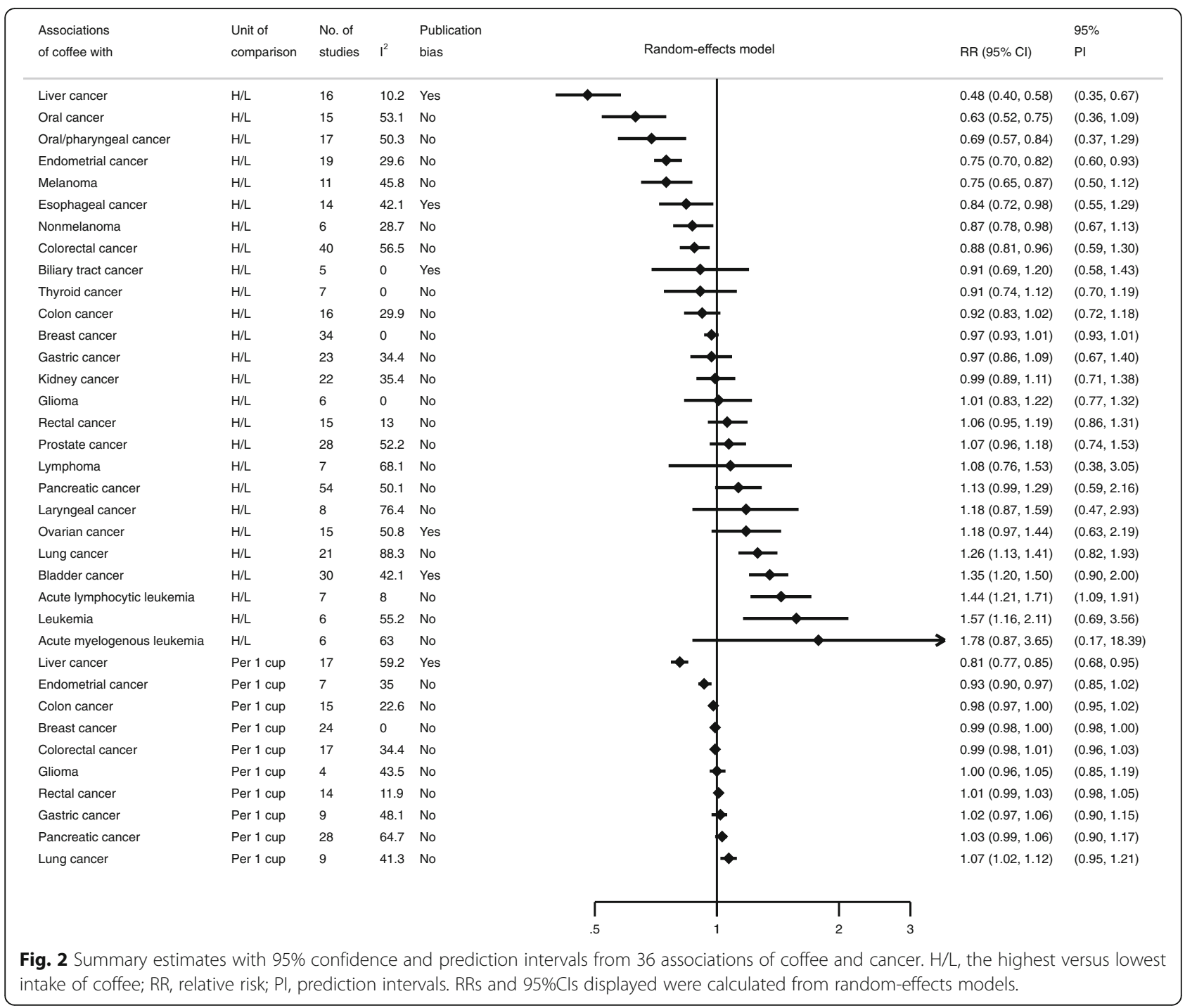

present for three outcomes, including liver cancer (per 1 cup), endometrial cancer (per 1 cup) and esophageal cancer $(\mathrm{H} / \mathrm{L})$. Using summary estimates of fixed or random-effects as plausible effect sizes gave similar results. There were 21 associations that included 10 or more studies. Six of 36 had evidence of publication bias using Egger's test.

\section{Evaluating the evidence}

Based on the above analyses, the association of childhood acute lymphocytic leukemia was supported by strong evidence in the current analyses (Table 3). For the highest versus lowest categories, six meta-analyses $(23.1 \%, 6 / 26)$ were supported by highly suggestive evidence for an association. Coffee intake was inversely related to the risk of endometrial cancer, liver cancer, melanoma, oral cancer, and oral/pharyngeal cancer while positively related to the risk of bladder cancer. The association between coffee and lung cancer was categorized as suggestive evidence. Four meta-analyses (15\%, 4/26) were supported by weak evidence including colorectal cancer, nonmelanoma, esophageal cancer, and leukemia. For dose-response analysis, only liver and endometrial cancers were judged as highly suggestive evidence. Three cancers were considered as having weak evidence in relation to coffee drinking including cancers of the colon, breast (women), and lung.

The AMSTAR 2 instrument adopted the rating process based on the identification of critical domains and distinguished the reviews into four categories: high, moderate, low, and extremely low. Twenty-six out of 28 reviews (93\%) scored as extremely low (online Additional file 1: Table S3). The low scores may be due to two main domains. First, none of the included reviews contained an explicit statement or published protocol 
Table 3 Summary of evidence grading for meta-analyses associating coffee intake with cancer incidence Level of evidence

\begin{tabular}{|c|c|c|c|}
\hline & Criteria used & Decreased risk & Increased risk \\
\hline Strong & $\begin{array}{l}P \text { in random-effects model }<=0.001 \\
\text { Number of cases }>1000 \\
I^{2}<=50 \% \\
95 \% \text { predictive intervals exclude the null value } \\
\text { Small study effects } P>0.1 \\
\text { Excess significance bias } P>0.1\end{array}$ & None & $\begin{array}{l}\text { Acute lymphocytic } \\
\text { leukemia }(H / L)\end{array}$ \\
\hline Highly suggestive & $\begin{array}{l}P \text { in random-effects model }<=0.001 \\
\text { Number of cases }>1000 \\
I^{2}<=75 \%\end{array}$ & $\begin{array}{l}\text { Endometrial cancer }(H / L) \text {; Endometrial cancer } \\
\text { (Per } 1 \text { cup); Liver cancer }(H / L) \text {; Liver cancer } \\
\text { (Per } 1 \text { cup); Melanoma }(H / L) ; \text { Oral cancer } \\
(H / L) \text {; Oral/pharyngeal cancer }(H / L)\end{array}$ & Bladder $(H / L)$ \\
\hline Suggestive & $\begin{array}{l}P \text { in random-effects model }<=0.001 \\
\text { Number of cases }>500\end{array}$ & None & Lung cancer $(\mathrm{H} / \mathrm{L})$ \\
\hline Weak & $P$ in random-effects model $<=0.05$ & $\begin{array}{l}\text { Breast cancer (Per } 1 \text { cup); Colon cancer (Per } 1 \text { cup); } \\
\text { Colorectal cancer }(\mathrm{H} / \mathrm{L}) \text {; Esophageal cancer }(\mathrm{H} / \mathrm{L}) ; \\
\text { Nonmelanoma }(\mathrm{H} / \mathrm{L})\end{array}$ & $\begin{array}{l}\text { Leukemia (H/L); Lung } \\
\text { (Per } 1 \text { cup) }\end{array}$ \\
\hline No association & $P$ in random-effects model $>0.05$ & 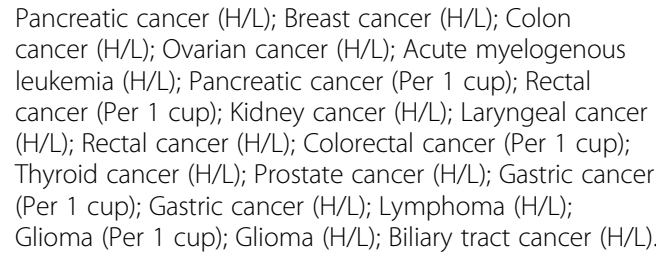 & \\
\hline
\end{tabular}

H/L Highest intake vs. lowest intake of coffee

prior to the conduct of the review. Second, none of the reviews searched the grey literature database.

\section{Secondary analysis}

When we included a meta-analysis of prospective cohort studies only, our main results were robust (online Additional file 1: Tables S4, S5, and S6). We still found highly suggestive evidence for the associations for liver and endometrial cancers. Several associations mainly based on case-control studies were not evaluated in sensitivity analyses. Associations for melanoma and lung cancer were considered as suggestive evidence. Other associations found in the main analysis were classified into weak evidence.

\section{Discussion}

In this umbrella review, we evaluated associations between coffee intake and 26 different cancer sites including 364,749 cancer cases. With the detailed evaluation of bias in the literature, our studies revealed that coffee intake was inversely associated with five cancer sites, namely, endometrial cancer, liver cancer, melanoma, oral cancer, and oral/pharyngeal cancer. For endometrial and liver cancers, there were robust evidences for a dosedependent association. Higher coffee intake was associated with an increased risk of childhood acute lymphocytic leukemia and bladder cancer. When we used a meta-analysis of prospective cohort studies, only associations for liver and endometrial cancers were further confirmed.
The International Agency for Research on Cancer (IARC) and the World Cancer Research Fund (WCRF) have judged the evidence of coffee intake and risk of cancer recently $[25,26]$. Their latest evaluation reported an inverse association with coffee drinking for liver and endometrial cancers. Consistent with this, the WCRF summarized the main findings from the Continuous Update Project (CUP) dose-response meta-analyses of cohort studies on coffee drinking and cancer risk reporting that it was probable that coffee intake was inversely associated with liver and endometrial cancers with low heterogeneity. The evidence was deemed suggestive for the risk of cancers of the mouth, pharynx and larynx, and of skin cancer. However, for other cancer sites, the evidence was too limited to draw a firm conclusion.

Our umbrella review of the existing evidence supports an inverse association between coffee intake and the risk of liver and endometrial cancers, which is similar to other studies [12, 27]. Previous studies only described the associations between coffee and liver and endometrial cancer and did not comprehensively evaluate the potential heterogeneity and bias. Our studies indicated there were still some questions before achieving a determinative conclusion. There was evidence of small study effects and excess significance bias for liver cancer. When we re-analyzed in meta-analyses of cohort studies, the results still tended to be affected by publication bias and excess significance bias. The association for endometrial cancer also had evidence of excess significance bias. These biases may degrade the evidence and lead to cautious and prudent conclusions. 
Our studies indicated higher coffee intake was inversely associated with melanoma and oral/pharyngeal cancer. However, these associations are mostly based on case-control studies that naturally tended to be affected by recall bias and selection bias. When we restricted to meta-analyses of cohort studies, the number of cases was less than 1000 for melanoma making it challenging to interpret the findings. More data from cohort studies will be needed to draw more firm conclusions for this cancer. For oral/pharyngeal cancer, substantial heterogeneity was found between cohort studies even though only four cohort studies were combined for oral cancer and six for oral/pharyngeal cancer. Similar to melanoma, associations of coffee with these rare cancers needs to be investigated in more cohorts or pooled studies.

The associations between coffee and cancers of bladder and lung have been highly debated $[13,28]$. In early reports by IARC, it was reported that there was a suggestive positive link between these two cancers with coffee based on case-control studies [29]. The current study indicated stronger evidence when included meta-analysis of all observational studies. However, these associations were categorized into weak or no evidence in metaanalyses of cohort studies. The recall bias and confounding effect of tobacco smoking may play a role in these observed associations. Recently, some debates are ignited about the acrylamide formed early in the roasting process of coffee beans, a carcinogen in both animals and human [30]. However, we did not have enough evidence to attribute the positive associations between coffee and these two types of cancers to the acrylamide because such a low exposure of acrylamide in coffee is unlikely to cause any cancers in human body [31,32].

Our study also indicated high coffee consumption during pregnancy was associated with acute lymphocytic leukemia in childhood based on a meta-analysis of six case-control studies. Even though the evidence is categorized as convincing evidence, we still need more cohort studies to illustrate the association between maternal coffee intake and childhood leukemia. For colon and breast cancers, if there is any association, coffee drinking may have a very small effect, which means these associations need a larger sample of population to be confirmed. For nonmelanoma and esophageal cancer, these associations were present mainly in case-control studies $[33,34]$. Therefore, more prospective studies are needed for a further conclusion.

For possible associations of coffee intake with the risk of cancer, the biological mechanism remains unclear. Numerous studies indicated that coffee drinking provides exposure to a range of biologically active chemicals, including caffeine and phenolic compounds that may impact health through various mechanisms, such as antioxidants, insulin sensitivity, liver function, and chronic inflammation [4, 35]. However, these benefits have not been obtained in randomized controlled studies (RCTs) yet. Therefore, to confirm these healthy effects and explore the underlying mechanism, new high quality RCTs with serum biomarker should be conducted.

Umbrella reviews rely on methodological quality and report transparency of the included meta-analyses [36]. Therefore, several limitations should be acknowledged in the current study. First, since umbrella reviews are observational studies, the reliability depended on the included meta-analysis directly and the original studies indirectly. It is impossible to control biases fielded in the original studies. Second, using the categorical association comparing the highest vs. lowest categories of coffee intake, or the linear association per one cup increase of coffee intake, may not reflect the real association (e.g. non-linear association) because we do not have the access to the individual-level data from the original studies. Finally, before a general recommendation on cancer prevention can be made, more researches are needed to improve understanding of how the volume and regularity of consumption, type of coffee, and style of preparation, such as adding milk or sugar, affect the risk of cancer.

\section{Conclusions}

Even though coffee has been associated with a lower risk of several common cancers in the literature, the associations for only liver cancer and endometrial cancer were supported by highly suggestive evidence. Findings for cancer at other sites were less consistent, presenting hints of uncertainty and/or bias, which need more confirmative studies in the future.

\section{Additional Files}

Additional file 1: Supplementary methods. Table S1. Excluded list through full-text review. Table S2. Description of meta-analyses of coffee consumption and cancer incidence with more than one meta-analysis. Table S3. AMSTAR score of included meta-analysis. Table S4. Description of meta-analyses only including cohort studies of coffee consumption and cancer incidence with more than one meta-analysis. Table S5. Description, evaluation of bias and heterogeneity in 34 associations of coffee intake and cancer incidence only including meta-analyses of cohort studies. Table S6. Summary of evidence grading for meta-analyses of cohort studies associating coffee intake and cancer incidence.

Additional file 2: Figure S1-S36. Forest plots of all observational studies. Figure S37-S70. Forest plots of cohort studies.

\section{Abbreviations}

Cl: Confidence interval; IARC: International agency for research on cancer; PI: Predictive interval; WCRF: World cancer research fund

\section{Acknowledgments}

We would appreciate the authors of the original meta-analysis for their contribution to this topic.

\section{Disclaimer}

Where authors are identified as personnel of the International Agency for Research on Cancer / World Health Organization, the authors alone are 
responsible for the views expressed in this article and they do not necessarily represent the decisions, policy or views of the International Agency for Research on Cancer / World Health Organization.

\section{Authors' contributions}

The authors' responsibilities were as follows: YBX obtained the funding; YBX and LGZ conceptualized the umbrella review and interpreted the data and results; LGZ, ZYL, and GSF conducted the research and performed the statistical analysis. LGZ drafted the initial manuscript; LGZ, ZYL, GSF, XWJ, YTT, $H L L, M J G$, and $Y B X$ substantively revised the manuscript and have approved the submitted version. YBX had final responsibility for the decision to submit the paper for publication.

\section{Funding}

This work was supported by grants of the National Key Project of Research and Development Program of China (2016YFC1302503), and the National Key Basic Research Program "973 Project" (2015CB554000). None of the funders had any influence on the study design; in the collection, analysis, and interpretation of data; in the writing of the report; and in the decision to submit the article for publication.

\section{Availability of data and materials}

No additional data are available.

\section{Ethics approval and consent to participate} Not applicable.

\section{Consent for publication}

Not applicable.

\section{Competing interests}

The authors declare that they have no competing interests.

\section{Author details}

${ }^{1}$ State Key Laboratory of Oncogene and Related Genes \& Department of Epidemiology, Shanghai Cancer Institute, Renji Hospital, Shanghai Jiaotong University School of Medicine, Shanghai, China. ${ }^{2}$ Section of Nutrition and Metabolism, International Agency for Research on Cancer, 150, cours Albert Thomas F-69372, Cedex 08 Lyon, France.

Received: 7 August 2019 Accepted: 20 January 2020

\section{Published online: 05 February 2020}

\section{References}

1. Cano-Marquina A, Tarin JJ, Cano A. The impact of coffee on health. Maturitas. 2013;75(1):7-21.

2. Park SY, Freedman ND, Haiman CA, Le Marchand L, Wilkens LR, Setiawan W. Prospective study of coffee consumption and cancer incidence in nonwhite populations. Cancer Epidemiol Biomark Prev. 2018;27(8):928-35.

3. Weinstein ND. Reactions to life-style warnings: coffee and cancer. Health Educ Q. 1985;12(2):129-34.

4. Bohn SK, Blomhoff R, Paur I. Coffee and cancer risk, epidemiological evidence, and molecular mechanisms. Mol Nutr Food Res. 2014;58(5):915-30.

5. Gaascht F, Dicato M, Diederich M. Coffee provides a natural multitarget pharmacopeia against the hallmarks of cancer. Genes Nutr. 2015;10(6):51.

6. Marrett LD, Walter SD, Meigs JW. Coffee drinking and bladder cancer in Connecticut. Am J Epidemiol. 1983;117(2):113-27.

7. Simon D, Yen S, Cole P. Coffee drinking and cancer of the lower urinary tract. J Natl Cancer Inst. 1975:54(3):587-91.

8. Bamia C, Lagiou P, Jenab M, Trichopoulou A, Fedirko V, Aleksandrova K, Pischon T, Overvad K, Olsen A, Tjonneland A, et al. Coffee, tea and decaffeinated coffee in relation to hepatocellular carcinoma in a European population: multicentre, prospective cohort study. Int J Cancer. 2015;136(8): 1899-908.

9. Caini S, Masala G, Saieva C, Kvaskoff M, Savoye I, Sacerdote C, Hemmingsson O, Hammer Bech B, Overvad K, Tjonneland A, et al. Coffee, tea and melanoma risk: findings from the European prospective investigation into Cancer and nutrition. Int J Cancer. 2017:140(10):2246-55.

10. Petrick JL, Freedman ND, Graubard BI, Sahasrabuddhe W, Lai GY, Alavanja MC, Beane-Freeman LE, Boggs DA, Buring JE, Chan AT, et al. Coffee consumption and risk of hepatocellular carcinoma and intrahepatic cholangiocarcinoma by sex: the liver cancer pooling project. Cancer Epidemiol Biomark Prev. 2015;24(9):1398-406.

11. Gunter MJ, Schaub JA, Xue X, Freedman ND, Gaudet MM, Rohan TE, Hollenbeck AR, Sinha R. A prospective investigation of coffee drinking and endometrial cancer incidence. Int J Cancer. 2012;131(4):E530-6.

12. Grosso G, Godos J, Galvano F, Giovannucci EL. Coffee, caffeine, and health outcomes: an umbrella review. Annu Rev Nutr. 2017;37:131-56.

13. Gruber K. Coffee consumption and bladder cancer are linked, analysis shows. BMJ. 2015;350:h1477.

14. Aromataris E, Fernandez R, Godfrey CM, Holly C, Khalil H, Tungpunkom P. Summarizing systematic reviews: methodological development, conduct and reporting of an umbrella review approach. Int J Evid Based Healthc. 2015;13(3):132-40.

15. Shamseer $L$, Moher $D$, Clarke $M$, Ghersi D, Liberati A, Petticrew M, Shekelle $P$, Stewart LA, Group P-P. Preferred reporting items for systematic review and meta-analysis protocols (PRISMA-P) 2015: elaboration and explanation. BMJ. 2015;350:g7647.

16. DerSimonian R, Laird N. Meta-analysis in clinical trials. Control Clin Trials. 1986;7(3):177-88.

17. Loannidis JP, Patsopoulos NA, Evangelou E. Uncertainty in heterogeneity estimates in meta-analyses. BMJ. 2007:335(7626):914-6.

18. Higgins JP, Thompson SG. Quantifying heterogeneity in a meta-analysis. Stat Med. 2002;21(11):1539-58.

19. Riley RD, Higgins JP, Deeks JJ. Interpretation of random effects metaanalyses. BMJ. 2011:342:d549.

20. Egger M, Davey Smith G, Schneider M, Minder C. Bias in meta-analysis detected by a simple, graphical test. BMJ. 1997;315(7109):629-34.

21. Ioannidis JPA. Clarifications on the application and interpretation of the test for excess significance and its extensions. J Math Psychol. 2013;57(5):184-7.

22. Bellou V, Belbasis L, Tzoulaki I, Evangelou E, loannidis JP. Environmental risk factors and Parkinson's disease: an umbrella review of meta-analyses. Parkinsonism Relat Disord. 2016;23:1-9.

23. Bellou V, Belbasis L, Tzoulaki I, Middleton LT, loannidis JPA, Evangelou E. Systematic evaluation of the associations between environmental risk factors and dementia: an umbrella review of systematic reviews and metaanalyses. Alzheimers Dement. 2017;13(4):406-18.

24. Markozannes G, Tzoulaki I, Karli D, Evangelou E, Ntzani E, Gunter MJ, Norat T, loannidis JP, Tsilidis KK. Diet, body size, physical activity and risk of prostate cancer: an umbrella review of the evidence. Eur J Cancer. 2016;69:61-9.

25. World Cancer Research Fund Network: Non-alcoholic drinks and the risk of cancer. In: Continuous Update Project. 2018.

26. International Agency for Research on Cancer Group: Evaluation of drinking coffee, maté, and very hot beverages. In: IARC Monographs. 2018.

27. Poole R, Kennedy OJ, Roderick P, Fallowfield JA, Hayes PC, Parkes J. Coffee consumption and health: umbrella review of meta-analyses of multiple health outcomes. BMJ. 2017;359:55024.

28. Sherman M. What is the relationship between coffee and lung cancer? Eur J Clin Nutr. 2016;70(9):1096.

29. World Health Organization: Coffee, Tea, Mate, Methylxanthines, and Methylglyoxal. In: IARC Monographs on the Evaluation of Carcinogenic Risks to Humans. Edited by Cancer IAfRo, vol. 2018; 1991.

30. Zhivagui M, Ng AWT, Ardin M, Churchwell MI, Pandey M, Renard C, Villar S, Cahais V, Robitaille A, Bouaoun L, et al. Experimental and pan-cancer genome analyses reveal widespread contribution of acrylamide exposure to carcinogenesis in humans. Genome Res. 2019;29(4):521-31.

31. Kotemori A, Ishihara J, Zha L, Liu R, Sawada N, Iwasaki M, Sobue T, Tsugane $\mathrm{S}$, Group JS. Dietary acrylamide intake and the risk of endometrial or ovarian cancers in Japanese women. Cancer Sci. 2018;109(10):3316-25.

32. Kotemori A, Ishihara J, Zha L, Liu R, Sawada N, Iwasaki M, Sobue T, Tsugane S, Group JS. Dietary acrylamide intake and risk of breast cancer: the Japan public health center-based prospective study. Cancer Sci. 2018;109(3):843-53.

33. Vaseghi G, Haghjoo-Javanmard S, Naderi J, Eshraghi A, Mahdavi M, Mansourian M. Coffee consumption and risk of nonmelanoma skin cancer: a dose-response meta-analysis. Eur J Cancer Prev. 2018;27(2): $164-70$.

34. Zheng JS, Yang J, Fu YQ, Huang T, Huang YJ, Li D. Effects of green tea, black tea, and coffee consumption on the risk of esophageal cancer: a systematic review and meta-analysis of observational studies. Nutr Cancer. 2013;65(1):1-16 
35. Ludwig IA, Clifford MN, Lean ME, Ashihara H, Crozier A. Coffee: biochemistry and potential impact on health. Food Funct. 2014;5(8): 1695-717.

36. Biondi-Zoccai G. Umbrella reviews in the evidence hierarchy. In: BiondiZoccai G, editor. Umbrella reviews: evidence synthesis with overviews of reviews and meta-epidemiologic studies. Latina, Italy: Sapienza University of Rome; 2016. p. 3-56.

\section{Publisher's Note}

Springer Nature remains neutral with regard to jurisdictional claims in published maps and institutional affiliations.

Ready to submit your research? Choose BMC and benefit from:

- fast, convenient online submission

- thorough peer review by experienced researchers in your field

- rapid publication on acceptance

- support for research data, including large and complex data types

- gold Open Access which fosters wider collaboration and increased citations

- maximum visibility for your research: over $100 \mathrm{M}$ website views per year

At BMC, research is always in progress.

Learn more biomedcentral.com/submissions 